\title{
The dynamics of a near-surface vortex in a two-layer ocean on the beta-plane
}

\author{
By E. S. BENILOV \\ Department of Mathematics, University of Limerick, Ireland \\ (Received 29 March 1999 and in revised form 4 March 2000)
}

The dynamics of a near-surface vortex are examined in a two-layer setting on the beta-plane. Initially, the vortex is radially symmetric and localized in the upper layer. Two non-dimensional parameters govern its evolution and translation: the ratio $\delta$ of the thickness of the vortex to the total depth of the fluid, and the non-dimensional beta-effect number $\alpha=\beta L / f$ ( $f$ and $\beta$ are the Coriolis parameter and its meridional gradient respectively, $L$ is the radius of the vortex). We assume, as suggested by oceanic observations, that $\alpha \ll \delta \ll 1$. A simple set of asymptotic equations is derived, which describes the beta-induced translation of the vortex and a dipolar perturbation developing on and under the vortex (in both layers).

This set was solved numerically for oceanic lenses, and the following features were observed: (i) The meridional (southward) component of the translation speed of the lens rapidly 'overtakes' the zonal (westward) component. The former grows approximately linearly, whereas the latter oscillates about the Nof (1981) value (i.e. about the speed of translation of a vortex in a one-layer reduce-gravity fluid). (ii) Vortices of the same shape, but different radii and amplitudes, follow the same trajectory. The amplitude and radius affect only the absolute value, but not the direction, of the translation speed. (iii) In the lower layer below the vortex, a 'region' is generated where the velocity of the fluid is growing linearly with time. The velocity field in the region becomes more and more homogeneous (and equal to the translation speed of the vortex).

\section{Introduction}

Much attention has been paid recently to the influence of the beta-effect upon oceanic vortices. The formulation of this problem is straightforward: what happens with an initially radially symmetric vortex on the beta-plane? In the one-layer setting, the answer to this question has been obtained in two parts. First, Nof (1981) demonstrated that warm-core near-surface lenses (i.e. vortices which outcrop onto the surface of the ocean) translate steadily westwards and calculated the speed of translation. Secondly, Benilov (1996) found the spatial structure of the translating lens and also extended all the results obtained to eddies (i.e. to vortices in a layer of non-zero background thickness). These steady solutions agree with oceanic observations, which indicate that vortices in the ocean exist for years.

It should be noted, however, that the (more realistic) baroclinic setting changes the problem quite significantly, as it does not support steadily translating monopole solutions (Flierl et al. 1983). Given that there is no reason to believe that the 'real' oceanic vortices have zero net angular momentum, the no net angular momentum theorem leads to the conclusion that they are non-steady. It is unclear though how 
long they remain coherent and spatially localized, and how the time scale of their evolution compares with the scale observed in the ocean (up to 2-3 years).

The first attempt to examine the evolution of vortices in a baroclinic ocean was made by Flierl (1984). As the present paper is an extension of Flierl's work, we shall describe the latter in some detail: it considered a near-surface lens surrounded by a layer of a heavier fluid, within the framework of the two-layer model. It was assumed, as suggested by oceanic observations, that the size of the lens is comparable to the Rossby radius $L_{R}$ associated with the thickness $H_{1}$ of the lens:

$$
L_{R}=\sqrt{g^{\prime} H_{1}} / f,
$$

where $g^{\prime}$ is the reduced acceleration due to gravity, and $f$ is the Coriolis parameter. The beta-induced motion was shown to depend on two non-dimensional parameters: the 'beta-effect number',

$$
\alpha=\beta L_{R} / f,
$$

where $\beta$ is the meridional gradient of the Coriolis parameter;

and the relative depth of the upper layer:

$$
\delta=H_{1} / H_{0},
$$

where $H_{0}$ is the total depth of the ocean.

In the 'real' ocean, both parameters are small. It is also important to know how they compare to each other. Flierl (1984) examined the case where

$$
\alpha \gtrsim \delta
$$

and put forward the following scheme:

(i) initially, Nof's (1981) mechanism makes the lens propagate westwards, inducing a flow around it in the lower layer;

(ii) then, the lower-layer motion generates Rossby waves, resulting in an energy flux directed away from the lens;

(iii) finally, the Rossby-wave field causes a drag force which makes the lens slowly drift equatorwards.

This simple and clear scheme appeals to one's physical intuition, but is extremely difficult to express in mathematical terms - and, as a result, relatively few quantitative predictions were obtained in Flierl's (1984) paper. Still, the above scheme was found useful in simpler oceanographic problems, the most recent example of which was considered by Swaters (1998), who was able to justify Flierl's scheme for a lens on a sloping bottom.

The main goal of the present paper is to examine the original problem formulated by Flierl (1984) and provide a consistent mathematical description for it. It should be noted, however, that assumption (1.3) contradicts oceanic observations, which suggest the opposite limit,

$$
\alpha \ll \delta
$$

(the typical oceanic values of $\alpha$ and $\delta$ are 0.01 and 0.1 , respectively). Flierl (1984) was aware of this contradiction, but considered (1.3) anyway, remarking that (1.4) 'appears to be a difficult limit to take'.

In the present paper, the more realistic limit (1.4) will be examined. The new assumption turns out to lead to new conclusions, the most unexpected of which seems to concern the unimportance of radiation. Interestingly, this can be established at an early stage of the analysis, immediately after the scaling $(\$ 2)$. In the absence of 


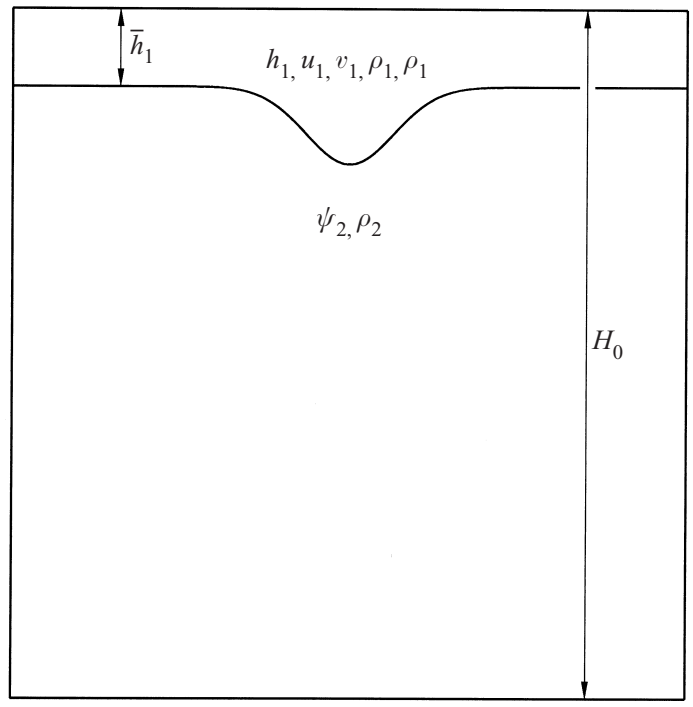

FIGURE 1. The formulation of the problem: a near-surface vortex in a two-layer ocean. Lenses correspond to $\bar{h}_{1}=0$.

radiation, the main mechanism causing the vortex to evolve is the direct interaction with the lower-layer flow, which it induces itself. As usual, the solution can be represented by a steadily translating monopole vortex with small dipolar corrections, and in $\S 3$, we shall derive a set of relatively simple equations describing the evolution of those corrections and translation of the vortex. These equations will be examined analytically and numerically $(\S 4)$.

\section{Scaling}

\subsection{Introductory remarks}

Consider a thin near-surface vortex (a lens or an eddy) on top of a thick layer of heavier fluid, on the beta-plane (see figure 1). The mathematical complexity of this problem is mainly due to the presence of three distinct time scales.

First, there is the advection time

$$
T_{0}=\frac{L}{U_{1}}
$$

where $L$ is the radius of the vortex and $U_{1}$ is the upper-layer velocity scale. Assuming that

$$
L=L_{R} \quad \text { and } \quad U_{1}=\sqrt{g^{\prime} H_{1}},
$$

which correspond to ageostrophic mesoscale motion, we obtain

$$
T_{0}=f^{-1} .
$$

It can be demonstrated that, at this time scale, the beta-effect changes the translation speed of the vortex from zero to, approximately, Nof's (1981) value, and adjusts the form of the vortex to Benilov's (1996) solution (this will be discussed in more detail later). As the flow in the lower layer is generated by the motion of the vortex, it develops at the same time scale, but is still too weak to affect the vortex. As a result, one can examine this stage of the evolution using the one-layer governing equations. 
Secondly, there is the time scale

$$
T_{\delta}=f^{-1} \delta^{-1},
$$

at which the growing flow in the lower layer begins to affect the vortex. Still, the lowerlayer flow is relatively weak, as it is generated by the beta-induced translation of the vortex and should coincide, in order of magnitude, with the speed of translation (i.e. with the Nof speed). This argument leads to the following estimate of the lower-layer velocity scale:

$$
U_{2}=\alpha \sqrt{g^{\prime} H_{1}} .
$$

Observe that, at this stage, the influence of the lower-layer flow (characterized by $U_{2} / U_{1}$ ) and the beta-effect are approximately of the same strength:

$$
\frac{U_{2}}{U_{1}}=\alpha .
$$

Thirdly, there is the time scale associated with the beta-effect,

$$
T_{\alpha}=f^{-1} \alpha^{-1} .
$$

In order to estimate the corresponding flow in the lower layer, we assume that $U_{2}$ keeps growing at the same rate as previously, which yields

$$
U_{2}=\delta \sqrt{g^{\prime} H_{1}} .
$$

This indicates that the flow in the lower layer has become, in a sense, stronger than the beta-effect:

$$
\frac{U_{2}}{U_{1}}=\delta \gg \alpha .
$$

It turns out, however (see $\S 4$ below), that the spatial structure of the lower-layer flow is such that it does not affect the zonal component of the translation speed (which continues to oscillate near the Nof (1981) value). However, the meridional component is affected, and soon the southward motion of the vortex becomes much faster than the westward motion:

$$
\frac{C_{x}}{C_{y}} \sim \frac{\alpha}{\delta},
$$

where $C_{x}$ and $C_{y}$ are the zonal and meridional components of the translation speed, respectively.

Strictly speaking, we should also introduce the time scale

$$
T_{\text {rad }}=f^{-1} \alpha^{-1} \delta^{-1},
$$

at which the Rossby-wave radiation in the lower layer affects the vortex (observe that, in the one-layer setting this scale would be $f^{-1} \alpha^{-1}$, but, in our problem, the flow in the lower layer is weak, hence the discriminating factor $\delta^{-1}$ ). As a result, all radiation-related effects are too slow to seriously affect the vortex.

The present paper is mainly devoted to step (ii) of the scheme in $\S 1$. We require that all faster processes $\left(\sim T_{0}\right)$ have died out, which is mathematically equivalent, as we shall see, to using the one-layer steady solution of Nof (1981) and Benilov (1996) as the initial condition. Thus, we assume that this solution is an attractor for any vortex - or we simply admit, for more incredulous readers, that we deal with a particular solution. The latter interpretation reduces the generality of the results obtained, but on the other hand allows one to clearly understand the limitations of the conclusions derived. 
To conclude this subsection, we mention that step (iii) of the above scheme is the most difficult mathematically, and no quantitative results will be presented on it here (this part of the work is in progress). We shall offer only some qualitative results in $\S 4.4$.

\subsection{Scaling}

We shall use Flierl's (1984) set of governing equations, which consists of the standard primitive equations for the flow in the upper layer (i.e. for the vortex):

$$
\begin{gathered}
\frac{\partial \boldsymbol{u}_{1}}{\partial t}+\boldsymbol{u}_{1} \cdot \nabla \boldsymbol{u}_{1}+\nabla p_{1}=(f+\beta y) \boldsymbol{u}_{1} \times \boldsymbol{k}, \\
\frac{\partial h_{1}}{\partial t}+\nabla \cdot\left(h_{1} \boldsymbol{u}_{1}\right)=0,
\end{gathered}
$$

and a quasi-geostrophic $(\mathrm{QG})$ equation for the lower layer:

$$
\frac{\partial}{\partial t}\left(\frac{f}{H_{0}} h_{1}+\nabla^{2} \psi_{2}\right)+\beta \frac{\partial \psi_{2}}{\partial x}+J\left(\psi_{2}, \frac{f}{H_{0}} h_{1}+\nabla^{2} \psi_{2}\right)=0,
$$

where $\boldsymbol{u}_{1}, p_{1}$, and $h_{1}$ are the velocity, pressure, and depth of the upper layer, $\boldsymbol{k}$ is the unit vector directed upwards, $\psi_{2}$ is the streamfunction of the lower layer, $(x, y)$ are the dimensional Cartesian coordinates, and $J(\psi, \phi)$ is the Jacobian operator. As $\psi_{2}$ is proportional to the lower-layer pressure:

$$
p_{2}=f \psi_{2},
$$

it is related to $p_{1}$ and $h_{1}$ by the hydrostatic condition:

$$
p_{1}=g^{\prime} h_{1}+f \psi_{2} \text {. }
$$

Note, that the QG approximation is legitimate for the lower layer because the displacement of the interface caused by the vortex is much smaller than the depth of the lower layer, and the beta-induced drift of the vortex is slow, hence the flow in the lower layer is weak and the corresponding Rossby number is small. We shall not discuss this matter in further detail, but refer the reader to the paper by Flierl (1984).

Next, we shall introduce the (beta-induced) displacement $(X(t), Y(t))$ of the vortex from its initial position and change the coordinate system such that its origin is linked to the vortex:

$$
x_{*}=x-X(t), \quad y_{*}=y-Y(t), \quad t_{*}=t .
$$

We rewrite equations (2.1)-(2.4) in terms of the new variables (asterisks omitted):

$$
\begin{gathered}
\left(\frac{\partial}{\partial t}-\dot{X} \frac{\partial}{\partial x}-\dot{Y} \frac{\partial}{\partial y}\right) u_{1}+u_{1} \frac{\partial u_{1}}{\partial x}+v_{1} \frac{\partial u_{1}}{\partial y}+\frac{\partial}{\partial x}\left(g^{\prime} h_{1}+f \psi_{2}\right)=(f+\beta y) v_{1}, \\
\left(\frac{\partial}{\partial t}-\dot{X} \frac{\partial}{\partial x}-\dot{Y} \frac{\partial}{\partial y}\right) v_{1}+u_{1} \frac{\partial v_{1}}{\partial x}+v_{1} \frac{\partial v_{1}}{\partial y}+\frac{\partial}{\partial y}\left(g^{\prime} h_{1}+f \psi_{2}\right)=-(f+\beta y) u_{1}, \\
\left(\frac{\partial}{\partial t}-\dot{X} \frac{\partial}{\partial x}-\dot{Y} \frac{\partial}{\partial y}\right) h_{1}+\frac{\partial}{\partial x}\left(h_{1} u_{1}\right)+\frac{\partial}{\partial y}\left(h_{1} v_{1}\right)=0 \\
\left(\frac{\partial}{\partial t}-\dot{X} \frac{\partial}{\partial x}-\dot{Y} \frac{\partial}{\partial y}\right)\left(\frac{f}{H_{0}} h_{1}+\nabla^{2} \psi_{2}\right)+\beta \frac{\partial \psi_{2}}{\partial x}+J\left(\psi_{2}, \frac{f}{H_{0}} h_{1}+\nabla^{2} \psi_{2}\right)=0 \\
\text { where } \dot{X}=\mathrm{d} X / \mathrm{d} t, \dot{Y}=\mathrm{d} Y / \mathrm{d} t
\end{gathered}
$$

\footnotetext{
where $\dot{X}=d X / d t, \dot{Y}=d Y / d t$.
} 
Next we shall introduce non-dimensional variables:

$$
\begin{gathered}
(\widetilde{x}, \widetilde{y})=(x / L, y / L), \quad \widetilde{t}=t / T, \quad(\tilde{\dot{X}}, \widetilde{Y})=\left(\dot{X} / C_{x}, \dot{Y} / C_{y}\right), \\
\left(\widetilde{u}_{1}, \widetilde{v}_{1}\right)=\left(u_{1} / U_{1}, v_{1} / U_{1}\right), \quad \widetilde{h}_{1}=h / H_{1}, \quad \widetilde{\psi}_{2}=\psi_{2} / \Psi_{2},
\end{gathered}
$$

where $L, T, C_{x, y}, U_{1}, H_{1}$, and $\Psi_{2}$ are the characteristic values of the corresponding variables. We shall assume that the radius of the vortex is comparable to the Rossby radius,

$$
L=\frac{\sqrt{g^{\prime} H_{1}}}{f},
$$

and that the Rossby number in the upper layer is of the order of unity, which leads to

$$
U_{1}=\sqrt{g^{\prime} H_{1}} .
$$

As mentioned above, we are interested in the medium-term evolution, i.e.

$$
T=\delta^{-1} f^{-1},
$$

when the translation speed of the vortex is comparable to the Nof speed, which leads to

$$
C_{x}=C_{y}=\alpha \sqrt{g^{\prime} H_{1}} .
$$

It can be demonstrated that we are now left with no other possibility, but to put

$$
\Psi_{2}=\alpha \frac{g^{\prime} H_{1}}{f},
$$

otherwise the leading order of the governing equations would become inconsistent for the most interesting case

$$
1 \gg \delta \gg \alpha .
$$

Substituting (2.9)-(2.15) into (2.5)-(2.8) and omitting all tildes and subscripts, we obtain

$$
\begin{gathered}
{\left[\delta \frac{\partial}{\partial t}-\alpha\left(\dot{X} \frac{\partial}{\partial x}+\dot{Y} \frac{\partial}{\partial y}\right)\right] u+u \frac{\partial u}{\partial x}+v \frac{\partial u}{\partial y}+\frac{\partial}{\partial x}(h+\alpha \psi)=(1+\alpha y) v,} \\
{\left[\delta \frac{\partial}{\partial t}-\alpha\left(\dot{X} \frac{\partial}{\partial x}+\dot{Y} \frac{\partial}{\partial y}\right)\right] v+u \frac{\partial v}{\partial x}+v \frac{\partial v}{\partial y}+\frac{\partial}{\partial y}(h+\alpha \psi)=-(1+\alpha y) u,} \\
{\left[\delta \frac{\partial}{\partial t}-\alpha\left(\dot{X} \frac{\partial}{\partial x}+\dot{Y} \frac{\partial}{\partial y}\right)\right] h+\frac{\partial}{\partial x}(h u)+\frac{\partial}{\partial y}(h v)=0,} \\
{\left[\frac{\partial}{\partial t}-\frac{\alpha}{\delta}\left(\dot{X} \frac{\partial}{\partial x}+\dot{Y} \frac{\partial}{\partial y}\right)\right]\left(h+\frac{\alpha}{\delta} \nabla^{2} \psi\right)+\frac{\alpha^{2}}{\delta^{2}} \frac{\partial \psi}{\partial x}+\frac{\alpha}{\delta} J\left(\psi, h+\frac{\alpha}{\delta} \nabla^{2} \psi\right)=0 .}
\end{gathered}
$$

The most interesting feature of the scaled equations is the smallness of the term responsible for the Rossby-wave radiation in the lower layer (i.e. the term involving $\partial \psi / \partial x$ in equation (2.19)). As we shall see in the next section, it will have to be omitted (together with all other terms proportional to $\alpha^{2} / \delta^{2}$ ).

\section{Asymptotic analysis of the governing equations}

In the first subsection of this section, we shall derive a set of asymptotic equations describing the translation of the vortex and development of small dipolar circulation 
on and under the vortex (those not interested in the mathematical development can skip it). In the second subsection, we shall discuss the properties of the equations derived.

\subsection{Derivation of asymptotic equations}

Instead of two independent small parameters $\alpha$ and $\delta$, it is now convenient to introduce a single parameter $\varepsilon$ such that

$$
\delta=\varepsilon, \quad \alpha=\varepsilon^{2}
$$

(which agrees with the oceanic values of $\delta \approx 0.1$ and $\alpha \approx 0.01$ ). Next we shall introduce the polar coordinates:

$$
x=r \cos \theta, \quad y=r \sin \theta
$$

and the polar variables:

$$
u=U \cos \theta-V \sin \theta, \quad v=U \sin \theta+V \cos \theta, \quad h=H, \quad \psi=\Psi .
$$

Substitution of (3.1)-(3.3) into (2.16)-(2.19) yields

$$
\begin{aligned}
\varepsilon \frac{\partial U}{\partial t}+\left(U-\varepsilon^{2} \dot{X} \cos \theta-\varepsilon^{2} \dot{Y} \sin \theta\right) \frac{\partial U}{\partial r} & +\frac{1}{r}\left(V+\varepsilon^{2} \dot{X} \sin \theta-\varepsilon^{2} \dot{Y} \cos \theta\right)\left(\frac{\partial U}{\partial \theta}-V\right) \\
& +\frac{\partial}{\partial r}\left(H+\varepsilon^{2} \Psi\right)=\left(1+\varepsilon^{2} r \sin \theta\right) V, \\
\varepsilon \frac{\partial V}{\partial t}+\left(U-\varepsilon^{2} \dot{X} \cos \theta-\varepsilon^{2} \dot{Y} \sin \theta\right) \frac{\partial V}{\partial r} & +\frac{1}{r}\left(V+\varepsilon^{2} \dot{X} \sin \theta-\varepsilon^{2} \dot{Y} \cos \theta\right)\left(\frac{\partial V}{\partial \theta}+U\right) \\
+ & \frac{1}{r} \frac{\partial}{\partial \theta}\left(H+\varepsilon^{2} \Psi\right)=-\left(1+\varepsilon^{2} r \sin \theta\right) U,
\end{aligned}
$$

$\varepsilon r \frac{\partial H}{\partial t}+\frac{\partial}{\partial r}\left[r\left(U-\varepsilon^{2} \dot{X} \cos \theta-\varepsilon^{2} \dot{Y} \sin \theta\right) H\right]+\frac{\partial}{\partial \theta}\left[\left(V+\varepsilon^{2} \dot{X} \sin \theta-\varepsilon^{2} \dot{Y} \cos \theta\right) H\right]=0$,

$$
\begin{aligned}
\frac{\partial H}{\partial t}+\varepsilon\left[\frac{\partial \nabla^{2} \Psi}{\partial t}+\left(-\frac{1}{r} \frac{\partial \Psi}{\partial \theta}\right.\right. & -\dot{X} \cos \theta-\dot{Y} \sin \theta) \frac{\partial H}{\partial r} \\
& \left.+\frac{1}{r}\left(\frac{\partial \Psi}{\partial r}+\dot{X} \sin \theta-\dot{Y} \cos \theta\right) \frac{\partial H}{\partial \theta}\right]=O\left(\varepsilon^{2}\right)
\end{aligned}
$$

where

$$
\nabla^{2} \Psi=\frac{1}{r} \frac{\partial}{\partial r}\left(r \frac{\partial \Psi}{\partial r}\right)+\frac{1}{r^{2}} \frac{\partial^{2} \Psi}{\partial \theta^{2}}
$$

and $O\left(\varepsilon^{2}\right)$ on the right-hand side of (3.7) denotes the terms proportional to $\alpha^{2} / \delta^{2}$. Equations (3.4)-(3.7) should be supplemented by the usual boundary conditions at the centre of the vortex:

$$
U+\frac{\partial V}{\partial \theta}=0, \quad V-\frac{\partial U}{\partial \theta}=0, \quad \frac{\partial H}{\partial \theta}=0, \quad \frac{\partial \Psi}{\partial \theta}=0 \quad \text { at } \quad r=0,
$$

and at infinity:

$$
U, V \rightarrow 0, \quad H, \Psi \rightarrow \bar{H}, \bar{\Psi} \quad \text { as } \quad r \rightarrow \infty,
$$


where the constants $\bar{H}$ and $\bar{\Psi}$ are the background values of the upper-layer depth and streamfunction, respectively. The case of lenses (where the interface outcrops onto the surface of the ocean) is included in the consideration as the limiting case $\bar{H}=0$ (this approach provides a perfectly legitimate way to bypass derivation of the boundary condition at the outcropping).

Next we expand the solution in powers of $\varepsilon$ :

$$
\begin{aligned}
& (U, V, H)=(U, V, H)^{(0)}+\varepsilon(U, V, H)^{(1)}+\cdots, \\
& \Psi=\Psi^{(0)}+\cdots, \quad(\dot{X}, \dot{Y})=(\dot{X}, \dot{Y})^{(0)}+\cdots,
\end{aligned}
$$

and assume that the zero-order solution is radially symmetric and steady (i.e. we deal with a nearly steady, nearly circular vortex):

$$
U^{(0)}=0, \quad V^{(0)}=V^{(0)}(r), \quad H^{(0)}=H^{(0)}(r) .
$$

Equations (3.4)-(3.6) yield, as they should, the requirement that any steady vortex be in the state of cyclostrophic balance:

$$
\frac{\mathrm{d} H}{\mathrm{~d} r}=V+\frac{1}{r} V^{2},
$$

where the superscript ${ }^{(0)}$ has been omitted.

Now when we assumed that the zero-order solution is steady, it becomes evident that the largest perturbation to equations (3.4)-(3.6) is $O\left(\varepsilon^{2}\right)$, hence

$$
U^{(1)}=V^{(1)}=H^{(1)}=0 .
$$

For the second-order solution, equations (3.4)-(3.7) yield

$$
\begin{gathered}
\frac{1}{r} V\left(\frac{\partial U^{(2)}}{\partial \theta}-V^{(2)}\right)+\frac{1}{r}\left(V^{(2)}+\dot{X}^{(0)} \sin \theta-\dot{Y}^{(0)} \cos \theta\right)(-V) \\
+\frac{\partial}{\partial r}\left(H^{(2)}+\Psi^{(0)}\right)=V^{(2)}+(r \sin \theta) V \\
\left(U^{(2)}-\dot{X}^{(0)} \cos \theta-\dot{Y}^{(0)} \sin \theta\right) \frac{\mathrm{d} V}{\mathrm{~d} r}+\frac{1}{r} V\left(\frac{\partial V^{(2)}}{\partial \theta}+U^{(2)}\right)+\frac{1}{r} \frac{\partial}{\partial \theta}\left(H^{(2)}+\Psi^{(0)}\right)=-U^{(2)}, \\
\frac{\partial}{\partial r}\left[r\left(U^{(2)}-\dot{X}^{(0)} \cos \theta-\dot{Y}^{(0)} \sin \theta\right) H\right] \\
+\frac{\partial}{\partial \theta}\left[V H^{(2)}+\left(V^{(2)}+\dot{X}^{(0)} \sin \theta-\dot{Y}^{(0)} \cos \theta\right) H\right]=0 \\
\frac{\partial \nabla^{2} \Psi^{(0)}}{\partial t}+\left(-\frac{1}{r} \frac{\partial \Psi^{(0)}}{\partial \theta}-\dot{X}^{(0)} \cos \theta-\dot{Y}^{(0)} \sin \theta\right) \frac{\partial H}{\partial r}=0 .
\end{gathered}
$$

As always, the first non-zero correction to a monopole vortex represents a weak dipolar circulation, in accordance with which we can separate $r$ and $\theta$ as follows:

$$
\begin{array}{ll}
U^{(2)}=\Phi_{c}(r, t) \cos \theta+\Phi_{s}(r, t) \sin \theta, & V^{(2)}=\Lambda_{c}(r, t) \cos \theta+\Lambda_{s}(r, t) \sin \theta, \\
H^{(2)}=\Gamma_{c}(r, t) \cos \theta+\Gamma_{s}(r, t) \sin \theta, & \Psi^{(0)}=P_{c}(r, t) \cos \theta+P_{s}(r, t) \sin \theta .
\end{array}
$$

Substitution of (3.14)-(3.15) into (3.10)-(3.13) and straightforward calculations yield 
(the superscript ${ }^{(0)}$ is omitted from $\dot{X}^{(0)}$ and $\dot{Y}^{(0)}$ ):

$$
\begin{gathered}
\frac{1}{r} V\left(\Phi_{s}-\Lambda_{c}\right)-\frac{1}{r}\left(\Lambda_{c}-\dot{Y}\right) V+\frac{\partial}{\partial r}\left(\Gamma_{c}+P_{c}\right)=\Lambda_{c} \\
\frac{1}{r} V\left(-\Phi_{c}-\Lambda_{s}\right)-\frac{1}{r}\left(\Lambda_{s}+\dot{X}\right) V+\frac{\partial}{\partial r}\left(\Gamma_{s}+P_{s}\right)=\Lambda_{s}+r V \\
\left(\Phi_{c}-\dot{X}\right) \frac{\mathrm{d} V}{\mathrm{~d} r}+\frac{1}{r} V\left(\Lambda_{s}+\Phi_{c}\right)+\frac{1}{r}\left(\Gamma_{s}+P_{s}\right)=-\Phi_{c} \\
\left(\Phi_{s}-\dot{Y}\right) \frac{\mathrm{d} V}{\mathrm{~d} r}+\frac{1}{r} V\left(-\Lambda_{c}+\Phi_{s}\right)-\frac{1}{r}\left(\Gamma_{c}+P_{c}\right)=-\Phi_{s} \\
\frac{\partial}{\partial r}[r(-\dot{X}) H]+\left[V \Gamma_{s}+\left(\Lambda_{s}+\dot{X}\right) H\right]=0 \\
\frac{\partial}{\partial r}\left[r\left(\Phi_{s}-\dot{Y}\right) H\right]-\left[V \Gamma_{c}+\left(\Lambda_{c}+\dot{Y}\right) H\right]=0 \\
\frac{\partial}{\partial t}\left[\frac{1}{r} \frac{\partial}{\partial r}\left(r \frac{\partial P_{c}}{\partial r}\right)-\frac{1}{r^{2}} P_{c}\right]-\left(\frac{1}{r} P_{s}+\dot{X}\right) \frac{\mathrm{d} H}{\mathrm{~d} r}=0 \\
\frac{\partial}{\partial t}\left[\frac{1}{r} \frac{\partial}{\partial r}\left(r \frac{\partial P_{s}}{\partial r}\right)-\frac{1}{r^{2}} P_{s}\right]-\left(-\frac{1}{r} P_{c}+\dot{Y}\right) \frac{\mathrm{d} H}{\mathrm{~d} r}=0
\end{gathered}
$$

The boundary conditions (3.8), (3.9) become

$$
\begin{gathered}
\Phi_{c}+\Lambda_{s}=0, \quad \Lambda_{c}-\Phi_{s}=0, \quad \Gamma_{c, s}=0, \quad P_{c, s}=0 \quad \text { at } \quad r=0, \\
(\Phi, \Lambda, \Gamma, P)_{c, s} \rightarrow 0 \quad \text { as } r \rightarrow \infty .
\end{gathered}
$$

System (3.16)-(3.25) is composed of an initial-value problem for $P_{c, s}(r, t),(3.22)-(3.23)$, and two boundary-value problems for $\left(\Phi_{c}, \Lambda_{s}, \Gamma_{s}\right)$ and $\left(\Phi_{s}, \Lambda_{c}, \Gamma_{c}\right):(3.17),(3.18),(3.20)$ and (3.16), (3.19), (3.21), respectively. The initial-value problem depends on the boundary-value problems only through the components $\dot{X}$ and $\dot{Y}$ of the translation speed (see the schematic in figure 2). $\dot{X}$ and $\dot{Y}$ are also unknown-they should be determined as eigenvalues of the corresponding boundary-value problems (it is shown how this can be done below). It turns out, however, that all the unknowns but $P_{c, s}$ can be eliminated from the governing equations!

In order to eliminate $\left(\Phi_{c}, \Lambda_{s}, \Gamma_{s}\right)$ and relate $\dot{X}$ to $P_{s}$, we rewrite equations (3.17), (3.18), and (3.20) as follows:

$$
\begin{gathered}
-V \Phi_{c}-(r+2 V) \Lambda_{s}+r \frac{\partial \Gamma_{s}}{\partial r}=r^{2} V+\dot{X} V-r \frac{\partial P_{s}}{\partial r}, \\
\left(r+V+r \frac{d V}{d r}\right) \Phi_{c}+V \Lambda_{s}+\Gamma_{s}=\dot{X} r \frac{\mathrm{d} V}{\mathrm{~d} r}-P_{s}, \\
\frac{\partial}{\partial r}\left(r H \Phi_{c}\right)+H \Lambda_{s}+V \Gamma_{s}=\dot{X} r \frac{\mathrm{d} H}{\mathrm{~d} r} .
\end{gathered}
$$

This is a system of linear inhomogeneous ODEs for $\left(\Phi_{c}, \Lambda_{s}, \Gamma_{s}\right)$, and it has a solution only if the vector of right-hand sides is orthogonal to the solutions of the adjoint homogeneous boundary-value problem (if any). Omitting the details, we note that the (unique) adjoint solution is

$$
\Phi_{c}^{+}=H, \quad \Lambda_{s}^{+}=H, \quad \Gamma_{s}^{+}=r+V .
$$




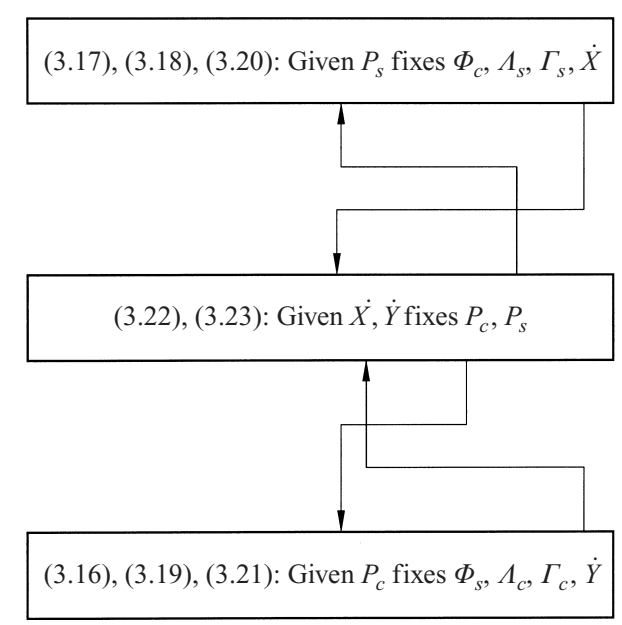

FIGURE 2. The structure of set (3.16)-(3.23).

One does not need to verify this (by inspection or otherwise); it is sufficient to just consider the following expression:

$$
\int_{0}^{\infty}[(3.26) \times H+(3.27) \times H+(3.28) \times(r+V)] \mathrm{d} r,
$$

then integrate it by parts, use the boundary conditions (3.24)-(3.25), and eventually make sure that $\Phi_{c}, \Lambda_{s}$, and $\Gamma_{s}$ disappear from the resulting relationship. We end up with a formula relating $\dot{X}$ to $P_{s}$ :

$$
\dot{X}=-\int_{0}^{\infty}\left(r P_{s} \frac{\mathrm{d} H}{\mathrm{~d} r}+r^{2} V H\right) \mathrm{d} r / \int_{0}^{\infty} r^{2} \frac{\mathrm{d} H}{\mathrm{~d} r} \mathrm{~d} r .
$$

Following a similar procedure for $\Phi_{s}, \Lambda_{c}$, and $\Gamma_{c}$, one can obtain a relationship for $\dot{Y}$ and $P_{c}$ :

$$
\dot{Y}=\int_{0}^{\infty} r P_{c} \frac{\mathrm{d} H}{\mathrm{~d} r} d r / \int_{0}^{\infty} r^{2} \frac{\mathrm{d} H}{\mathrm{~d} r} \mathrm{~d} r .
$$

Equations (3.22) and (3.23) are equivalent to the corresponding expressions, (13) and (15), of (Flierl 1984), despite the fact that those were obtained for a different limit $(\alpha \gtrsim \delta)$.

Equations (3.22)-(3.23) and (3.29)-(3.30) form a closed system for $P_{c}, P_{s}, \dot{X}$, and $\dot{Y}$.

\subsection{Discussion}

Rewrite equations (3.22)-(3.23), (3.29)-(3.30) in the form

$$
\begin{gathered}
\frac{\partial}{\partial t}\left[\frac{\partial}{\partial r}\left(r \frac{\partial P_{c}}{\partial r}\right)-\frac{1}{r} P_{c}\right]=\frac{\mathrm{d} H}{\mathrm{~d} r}\left(r \dot{X}+P_{s}\right), \\
\frac{\partial}{\partial t}\left[\frac{\partial}{\partial r}\left(r \frac{\partial P_{s}}{\partial r}\right)-\frac{1}{r} P_{s}\right]=\frac{\mathrm{d} H}{\mathrm{~d} r}\left(r \dot{Y}-P_{c}\right), \\
\dot{X}=\frac{1}{M} \int_{0}^{\infty} r \frac{\mathrm{d} H}{\mathrm{~d} r} P_{s} \mathrm{~d} r+\frac{A}{M} \\
\dot{Y}=-\frac{1}{M} \int_{0}^{\infty} r \frac{\mathrm{d} H}{\mathrm{~d} r} P_{c} \mathrm{~d} r
\end{gathered}
$$


where

$$
A=\int_{0}^{\infty} r^{2} V H \mathrm{~d} r, \quad M=-\int_{0}^{\infty} r^{2} \frac{\mathrm{d} H}{\mathrm{~d} r} \mathrm{~d} r .
$$

are the non-dimensional net angular momentum and mass anomaly of the vortex, respectively (recall that $V(r)$ and $H(r)$ are the swirl velocity and thickness of the vortex). We shall use the simplest initial condition

$$
P_{c}=P_{s}=0, \quad X=Y=0 \quad \text { at } \quad t=0,
$$

i.e. the evolution starts from a radially symmetric, unperturbed vortex. The boundary conditions (3.24)-(3.25) yield

$$
P_{c}=P_{s}=0 \quad \text { as } \quad r \rightarrow 0, \infty .
$$

(i) Consider the following combination of the governing equations:

$$
\int_{0}^{\infty}\left[(3.31) \times P_{c}-(3.32) \times P_{s}\right] \mathrm{d} r .
$$

Integrating by parts and using the boundary conditions, we obtain

$$
\begin{aligned}
- & \frac{1}{2} \frac{\mathrm{d}}{\mathrm{d} t} \int_{0}^{\infty}\left[r\left(\frac{\partial P_{c}}{\partial r}\right)^{2}+\frac{1}{r} P_{c}^{2}+r\left(\frac{\partial P_{s}}{\partial r}\right)^{2}+\frac{1}{r} P_{s}^{2}\right] \mathrm{d} r \\
& =\dot{X} \int_{0}^{\infty} r \frac{\mathrm{d} H}{\mathrm{~d} r} P_{c} \mathrm{~d} r+\int_{0}^{\infty} \frac{\mathrm{d} H}{\mathrm{~d} r} P_{s} P_{c} \mathrm{~d} r+\dot{Y} \int_{0}^{\infty} r \frac{\mathrm{d} H}{\mathrm{~d} r} P_{s} \mathrm{~d} r-\int_{0}^{\infty} \frac{\mathrm{d} H}{\mathrm{~d} r} P_{c} P_{s} \mathrm{~d} r
\end{aligned}
$$

Then take into account (3.33), (3.34), integrate with respect to $t$, and use the initial conditions to obtain

$$
\frac{1}{2} \int_{0}^{\infty}\left[r\left(\frac{\partial P_{c}}{\partial r}\right)^{2}+\frac{1}{r} P_{c}^{2}+r\left(\frac{\partial P_{s}}{\partial r}\right)^{2}+\frac{1}{r} P_{s}^{2}\right] \mathrm{d} r=A Y .
$$

The left-hand side of this identity is non-negative (it represents, in fact, the kinetic energy of the lower-layer flow), hence anticyclones (warm-core vortices, for which $A<0$ ) drift southwards $(Y \leqslant 0)$, whereas cyclones drift northwards. Invariant (3.38) will be used later to monitor the accuracy of simulation of our equations.

(ii) Observe that at $t=0$, the zero initial condition for the lower layer reduces the speed of the vortex to the non-dimensional Nof (1981) value:

$$
\dot{X}=\frac{A}{M}, \quad \dot{Y}=0,
$$

and also eliminates the effect of the lower layer from the upper-layer equations (3.16)(3.21) (which then coincide with the corresponding one-layer equations of Benilov (1996)). Thus, the initial condition for our asymptotic equations describes the steadily translating one-layer vortex. This implies that we have 'skipped' the first stage of the evolution of the vortex, when it adjusts its speed and shape to the beta-effect. Mathematically, this occurred due to the requirement that the time scale of the motion be slow, which made the time-derivative terms in the upper-layer equations negligible.

(iii) One should also be very careful in applying our results to eddies. In contrast to lenses, for which the upper-layer dipolar flow always remains bounded, the case of eddies is much more complicated. As demonstrated by Benilov (1996), most cyclonic eddies have their speeds of translation inside the range of allowable Rossby waves and the beta-effect destroys them through radiation. Mathematically, this is reflected 


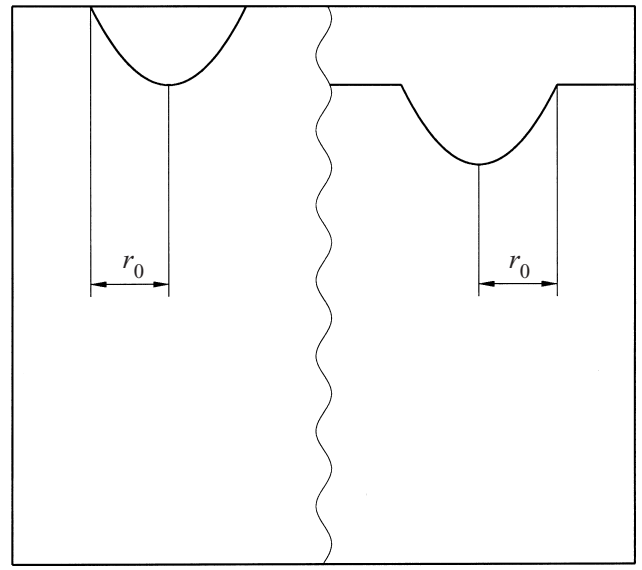

FIGURE 3. A lens (left) and an eddy with compact support (right).

by the unboundedness of the upper-layer dipolar solution:

$$
\Phi_{c, s}, \Lambda_{c, s}, \Gamma_{c, s}=O(r) \quad \text { as } r \rightarrow \infty
$$

(the orthogonality of the right-hand sides of equations (3.26)-(3.28) to the adjoint solution has removed the exponential, but not the linear, growth of $\left.\left(\Phi_{c}, \Lambda_{s}, \Gamma_{s}\right)\right)$. However, anticyclonic vortices (of which lenses can be treated as a limiting case) translate faster than the fastest Rossby wave and are thus unaffected by the radiation.

In what follows, it is implied that we consider only anticyclonic vortices.

\section{Eddies with compact support and lenses}

In this section we shall concentrate on eddies with compact support and lenses, i.e. on vortices for which

$$
\frac{\mathrm{d} H}{\mathrm{~d} r}=0 \quad \text { if } \quad r \geqslant r_{0},
$$

where $r_{0}$ is the radius of the vortex (see the schematic in figure 3). For the sake of brevity, both types of vortices will be referred to as 'lenses'.

\subsection{Conditions at the boundary of the lens}

Although we assumed that there is no radially symmetric monopolar flow on the outside of the lens, it does not mean that the dipolar circulation also stays inside. In order to find the structure of the flow for $r \geqslant r_{0}$, we multiply (3.31) and (3.32) by $r$ and integrate them with respect to $r$, from 0 to $r$. After straightforward calculations involving integration by parts we obtain

$$
\begin{aligned}
& r \frac{\partial}{\partial t}\left(r \frac{\partial P_{c}}{\partial r}-P_{c}\right)=\int_{0}^{r} r^{\prime} \frac{\mathrm{d} H\left(r^{\prime}\right)}{\mathrm{d} r^{\prime}}\left[r^{\prime} \dot{X}+P_{s}\left(r^{\prime}\right)\right] \mathrm{d} r^{\prime}, \\
& r \frac{\partial}{\partial t}\left(r \frac{\partial P_{s}}{\partial r}-P_{s}\right)=\int_{0}^{r} r^{\prime} \frac{\mathrm{d} H\left(r^{\prime}\right)}{\mathrm{d} r^{\prime}}\left[r^{\prime} \dot{Y}-P_{c}\left(r^{\prime}\right)\right] \mathrm{d} r^{\prime} .
\end{aligned}
$$

For the region on the outside of the lens, we then obtain

$$
r \frac{\partial}{\partial t}\left(r \frac{\partial P_{c}}{\partial r}-P_{c}\right)=-M \dot{X}+\int_{0}^{r_{0}} r^{\prime} \frac{\mathrm{d} H\left(r^{\prime}\right)}{\mathrm{d} r^{\prime}} P_{s}\left(r^{\prime}\right) \mathrm{d} r^{\prime} \quad \text { if } \quad r \geqslant r_{0},
$$




$$
r \frac{\partial}{\partial t}\left(r \frac{\partial P_{s}}{\partial r}-P_{s}\right)=-M \dot{Y}-\int_{0}^{r_{0}} r^{\prime} \frac{\mathrm{d} H\left(r^{\prime}\right)}{\mathrm{d} r^{\prime}} P_{c}\left(r^{\prime}\right) \mathrm{d} r^{\prime} \quad \text { if } \quad r \geqslant r_{0} .
$$

Substitution of (3.33) and (3.35) into these equations yields

$$
\begin{array}{cc}
r \frac{\partial}{\partial t}\left(r \frac{\partial P_{c}}{\partial r}-P_{c}\right)=-A & \text { if } \quad r \geqslant r_{0}, \\
r \frac{\partial}{\partial t}\left(r \frac{\partial P_{s}}{\partial r}-P_{s}\right)=0 & \text { if } \quad r \geqslant r_{0},
\end{array}
$$

which can be integrated with respect to $t$ using the initial condition (3.35), and then solved using the boundary condition at $r=\infty$ (see (3.37)):

$$
\begin{array}{cc}
P_{c}=\frac{A}{2 r} t \quad \text { if } \quad r \geqslant r_{0}, \\
P_{s}=0 \quad \text { if } \quad r \geqslant r_{0} .
\end{array}
$$

\subsection{Discussion}

The linearly growing boundary condition (4.1) has far-reaching consequences, as it somewhat limits the applicability of our asymptotic expansion. It should be clearly understood though, that numerical simulations (see below) demonstrate that this growth is relatively slow, and the error does not exceed $15 \%$ even for a relatively long-term evolution ( $\sim 6$ months). Also, all previous asymptotic theories for vortices influenced by weak beta-effect predict exactly the same linear growth at infinity (e.g. Reznik \& Dewar 1994 or Sutyrin \& Flierl 1994)†. This had not been noticed only because no-one considered lenses - instead, all authors considered eddies with 'tails' at $r \rightarrow \infty$, for which this growth is not apparent!

In order to illustrate this property of the beta-plane equations, consider the (simplest) model of one-layer QG dynamics examined previously by Reznik \& Dewar (1994). In the non-dimensional form, it is

$$
\frac{\partial \nabla^{2} \psi}{\partial t}+J\left(\psi, \nabla^{2} \psi\right)+\alpha \frac{\partial \psi}{\partial x}=0
$$

As usual, we change to the reference frame moving with the vortex:

$$
\frac{\partial \nabla^{2} \psi}{\partial t}-\dot{X} \frac{\partial \nabla^{2} \psi}{\partial x}-\dot{Y} \frac{\partial \nabla^{2} \psi}{\partial y}+J\left(\psi, \nabla^{2} \psi\right)+\alpha \frac{\partial \psi}{\partial x}=0 .
$$

Assuming the beta-effect to be weak $(\alpha \ll 1)$ and hence the beta-induced translation to be slow, we introduce

$$
\tilde{\dot{X}}=\dot{X} / \alpha, \quad \tilde{\dot{Y}}=\dot{Y} / \alpha .
$$

Substituting (4.5) into (4.4) and omitting tildes, we obtain

$$
\frac{\partial \nabla^{2} \psi}{\partial t}-\alpha \dot{X} \frac{\partial \nabla^{2} \psi}{\partial x}-\alpha \dot{Y} \frac{\partial \nabla^{2} \psi}{\partial y}+J\left(\psi, \nabla^{2} \psi\right)+\alpha \frac{\partial \psi}{\partial x}=0 .
$$

Next we expand the solution in powers of $\alpha$ :

$$
\psi=\psi^{(0)}+\alpha \psi^{(1)}+\cdots
$$

$\dagger$ This claim does not include the papers by Flierl (1984) and Swaters (1998), who considered the case of strong beta-effect, where the beta-term is included in the leading-order equations. 
where $\psi^{(0)}$ represents a steadily translating radially symmetric vortex:

$$
\psi^{(0)}=H(r)
$$

and $\psi^{(1)}$ represents the dipolar correction:

$$
\psi^{(1)}=P_{c}(r, t) \cos \theta+P_{s}(r, t) \sin \theta .
$$

Straightforward calculations yield

$$
\begin{array}{r}
\frac{\partial}{\partial t}\left[\frac{1}{r} \frac{\partial}{\partial r}\left(r \frac{\partial P_{c}}{\partial r}\right)-\frac{1}{r^{2}} P_{c}\right]+\frac{1}{r^{2}}\left[\frac{\mathrm{d} H}{\mathrm{~d} r} \frac{\partial}{\partial r}\left(r \frac{\partial P_{s}}{\partial r}\right)-P_{s} \frac{\mathrm{d}}{\mathrm{d} r}\left(r \frac{\mathrm{d}^{2} H}{\mathrm{~d} r^{2}}\right)\right] \\
=\frac{1}{r} \dot{X}\left[\frac{\mathrm{d}}{\mathrm{d} r}\left(r \frac{\mathrm{d}^{2} H}{\mathrm{~d} r^{2}}\right)-\frac{1}{r} \frac{\mathrm{d} H}{\mathrm{~d} r}\right]-\frac{\mathrm{d} H}{\mathrm{~d} r}, \\
\frac{\partial}{\partial t}\left[\frac{1}{r} \frac{\partial}{\partial r}\left(r \frac{\partial P_{s}}{\partial r}\right)-\frac{1}{r^{2}} P_{s}\right]-\frac{1}{r^{2}}\left[\frac{\mathrm{d} H}{\mathrm{~d} r} \frac{\partial}{\partial r}\left(r \frac{\partial P_{c}}{\partial r}\right)-P_{c} \frac{\mathrm{d}}{\mathrm{d} r}\left(r \frac{\mathrm{d}^{2} H}{\mathrm{~d} r^{2}}\right)\right] \\
=\frac{1}{r} \dot{Y}\left[\frac{\mathrm{d}}{\mathrm{d} r}\left(r \frac{\mathrm{d}^{2} H}{\mathrm{~d} r^{2}}\right)-\frac{1}{r} \frac{\mathrm{d} H}{\mathrm{~d} r}\right]
\end{array}
$$

(in deriving (4.6)-(4.7), we used the identity

$$
\frac{\mathrm{d}}{\mathrm{d} r}\left[\frac{1}{r} \frac{\mathrm{d}}{\mathrm{d} r}\left(r \frac{\mathrm{d} H}{\mathrm{~d} r}\right)\right]=\frac{1}{r} \frac{\mathrm{d}}{\mathrm{d} r}\left(r \frac{\mathrm{d}^{2} H}{\mathrm{~d} r^{2}}\right)-\frac{1}{r^{2}} \frac{\mathrm{d} H}{\mathrm{~d} r},
$$

which can be verified by inspection). Next we multiply (4.6) by $r^{2}$ and integrate with respect to $r$, from 0 to $r$. Straightforward calculations involving integration by parts yield

$r \frac{\partial}{\partial t}\left(r \frac{\partial P_{c}}{\partial r}-P_{c}\right)+r\left(\frac{\mathrm{d} H}{\mathrm{~d} r} \frac{\partial P_{s}}{\partial r}-P_{s} \frac{\mathrm{d}^{2} H}{\mathrm{~d} r^{2}}\right)=\dot{X} r\left(r \frac{\mathrm{d}^{2} H}{\mathrm{~d} r^{2}}-\frac{\mathrm{d} H}{\mathrm{~d} r}\right)-\int_{0}^{r} r^{\prime 2} \frac{\mathrm{d} H\left(r^{\prime}\right)}{\mathrm{d} r^{\prime}} \mathrm{d} r^{\prime}$.

For the region on the outside of an eddy with compact support, this can be reduced to

$$
r \frac{\partial}{\partial t}\left(r \frac{\partial P_{c}}{\partial r}-P_{c}\right)=M \quad \text { if } \quad r \geqslant r_{0}
$$

where, as before,

$$
M=-\int_{0}^{r_{0}} r^{2} \frac{\mathrm{d} H(r)}{\mathrm{d} r} \mathrm{~d} r
$$

is the net mass anomaly of the vortex. Equation (4.9) can be readily solved:

$$
P_{c}=-\frac{M}{2 r} t \quad \text { if } \quad r \geqslant r_{0},
$$

which is equivalent to (4.1) (it should be kept in mind here that, in the QG approximation $M \approx A$ ). If, however, the vortex has tails, i.e. if

$$
\frac{\mathrm{d} H}{\mathrm{~d} r} \neq 0 \quad \text { for all } r,
$$

one cannot reduce (4.8) to (4.9) and thus obtain solution (4.10). It does not, of course, mean that in this case the solution remains bounded as $t \rightarrow \infty$-it still grows, although not linearly. As demonstrated by Reznik \& Dewar (1994) for certain long-distance 
behaviours of $H(r)$, a proper choice of the translation velocity $(\dot{X}, \dot{Y})$ can reduce the growth to a logarithmic or slower-than-linear algebraic growth.

In conclusion of this subsection, we reiterate that the linear growth of the solution has little effect on the validity of our asymptotic method up to relatively large timescales - see the results of numerical modelling in the next subsection.

\subsection{Numerical solution}

In order to avoid solving our asymptotic equations in an unbounded domain, it is convenient to formulate a boundary conditions based on the outside-lens solution (4.1)-(4.2):

$$
P_{c}=\frac{A t}{2 r_{0}}, \quad P_{s}=0 \quad \text { at } \quad r=r_{0} .
$$

The resulting initial/boundary-value problem was solved using the second-order Runge-Kutta scheme for the time derivatives, and the Simpson integration routine for the inversion of the linear operators on the left-hand sides of (3.31) and (3.32). Invariant (3.38) was used to monitor the numerical accuracy (the non-conservation never exceeded $0.1 \%$ ). We considered two types of lens: the solid-body rotating vortex

$$
V(r)= \begin{cases}V_{\max }\left(r / r_{0}\right) & \text { if } \quad r<r_{0} \\ 0 & \text { if } \quad r \geqslant r_{0},\end{cases}
$$

and a differentially rotating vortex

$$
V(r)= \begin{cases}2 V_{\max }\left(r / r_{0}\right) \exp \left[\frac{3\left(4 r^{2}-r_{0}^{2}\right)}{8\left(r^{2}-r_{0}^{2}\right)}\right] & \text { if } r<r_{0} \\ 0 & \text { if } r \geqslant r_{0},\end{cases}
$$

where $V_{\max }$ is the maximum swirl velocity of the lens and $r_{0}$ is its size (see figure 4). Equation (4.11) was chosen because it is the simplest profile possible and has been used many times by other researchers. We shall use it to examine the theoretical properties of the equations derived, but ( 4.11) is not a very realistic model of oceanic lenses, as its outer 'slope' is infinitesimally thin. The differentially rotating vortex (4.12) is free from this shortcoming, as its parameters are adjusted in such a way that the maximum of $V(r)$ is located exactly at $r=\frac{1}{2} r_{0}$.

Our equations have only one spatial variable, and the processor time was not an issue - which enabled us to examine a wide range of parameters of lenses. We shall present here the most interesting results, which were obtained for

$$
0.55 \leqslant V_{\max } \leqslant 0.85 \mathrm{~m} \mathrm{~s}^{-1}, \quad 55 \leqslant \frac{1}{2} r_{0} \leqslant 70 \mathrm{~km},
$$

which represent medium sized, strong but not the strongest, oceanic rings. The parameters of the ocean in all cases were

$$
\frac{\rho_{2}-\rho_{1}}{\rho_{2}}=0.0005, \quad H_{0}=5000 \mathrm{~m}, \quad \text { latitude }=45^{\circ},
$$

where $\left(\rho_{2}-\rho_{1}\right) / \rho_{2}$ is the relative density difference, and $H_{0}$ is the total depth of the ocean. In all cases, we let the simulations run for 180 days.

We observed the following features of the evolution of lenses.

(i) As $t \rightarrow \infty$, the solution always approaches the following limiting regime:

$$
P_{s} \text { and } \dot{X} \text { remain bounded, } \quad P_{c} \rightarrow \frac{A t}{2 r_{0}^{2}} r, \quad \dot{Y} \rightarrow \frac{A t}{2 r_{0}^{2}} \quad \text { for } r<r_{0} \text {. }
$$

It can be readily verified that this regime is asymptotically consistent with the 

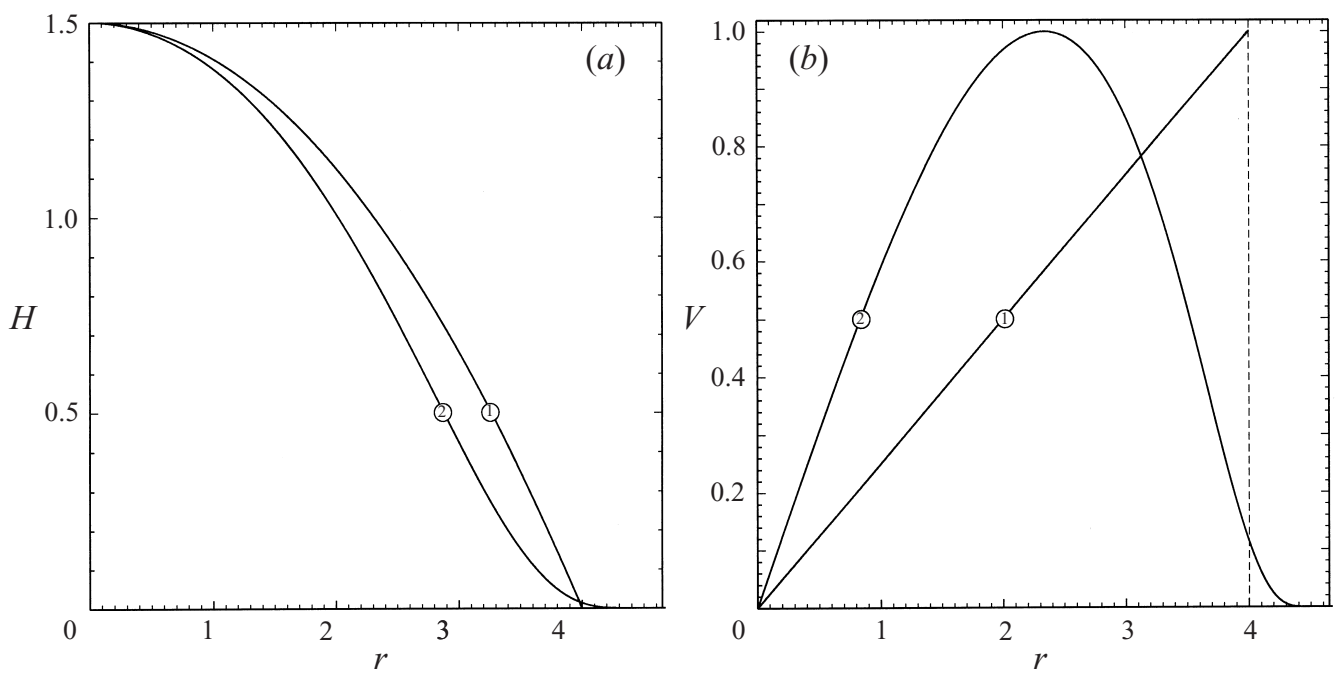

FIGURE 4. The profiles of lenses used in our simulations: (1) solid-body rotating lens (4.11); (2) differentially rotating lens (4.12). (a) Thickness; (b) swirl velocity.

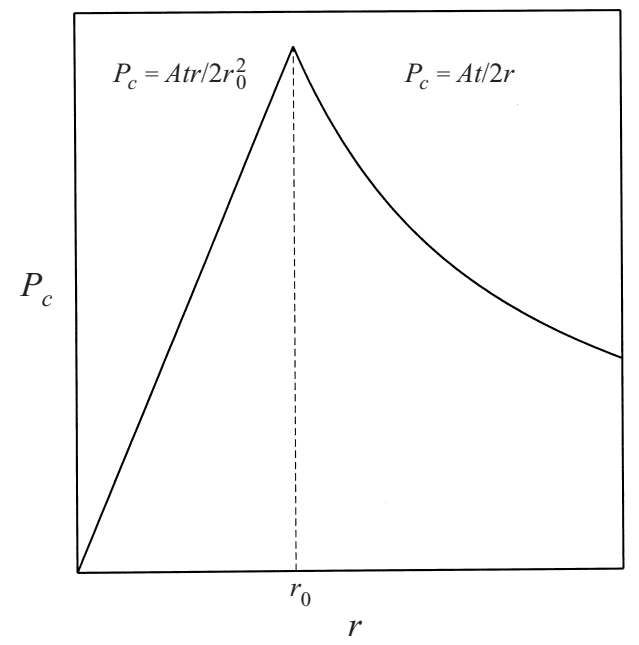

FIGURE 5. The limiting solution (4.13), (4.1).

governing equations. It implies, however, that $P_{c}$ has a 'spike' at $r=r_{0}$ (see figure 5). Clearly, the spike cannot develop from a smooth initial condition, which means that there must be a transitional region inside the lens, near its boundary (see figure 6).

The physical meaning of solution (4.13) becomes clear if we look at the lower-layer flow:

$$
\psi=P_{c} \cos \theta+P_{s} \sin \theta \approx \frac{A t}{2 r_{0}^{2}} r \cos \theta=\frac{A t}{2 r_{0}^{2}} x \Rightarrow v \approx \frac{A t}{2 r_{0}^{2}} .
$$

Thus, (4.13) describes a 'circle' of lower-layer fluid with meridional velocity constant in space and linearly growing in time. At the boundary of the circle, there is a transitional region which matches the circle to the flow around the lens.

The co-moving flow in the lower layer under the lens has a simple physical explanation. Recall that, in terms of the velocity field, the lens is initially localized in 


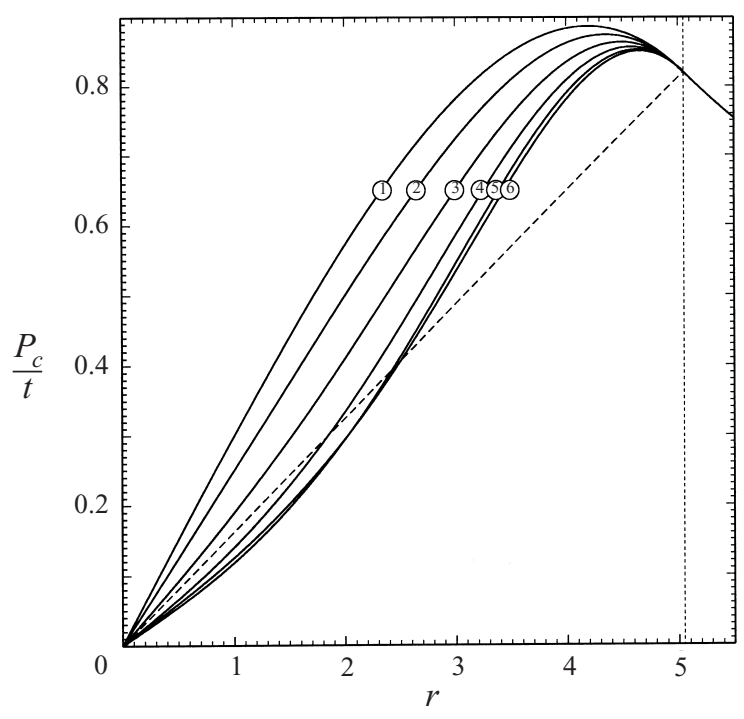

FIGURE 6. A typical evolution of the dipolar circulation associated with a solid-body rotating lens with $H_{\max }=400 \mathrm{~m}, r_{0}=120 \mathrm{~km}$. The solution is scaled by $t$ to 'remove' the linear growth; the dashed line shows the limiting solution (4.13); the dotted line shows the boundary of the lens; the labels of the curves show the number of months since the beginning of the evolution (all other variables are non-dimensional).

the upper layer:

$$
\psi_{2}=0 \quad \text { at } \quad t=0 .
$$

If, however, we look at the potential vorticity $(\mathrm{PV})$ of the lower layer,

$$
P V_{2}=\alpha \nabla^{2} \psi+\delta h+\alpha y,
$$

it becomes clear that the PV of particles under the lens (where $h \neq 0$ ) differs significantly from that of the 'outside' particles. As the displacement $h$ is caused by the lens and therefore translates with the lens, the particles below have to follow it otherwise they would not be able to preserve their anomalous PV values. Interestingly, the trapping of second-layer particles below the eddy has been noted within the framework of other vortex models - see the paper by Sutyrin \& Dewar (1992), who considered eddies of radius much larger than the deformation radius, in a setting similar to ours (two-layer fluid on the beta-plane).

Generally speaking, the growth of $\psi$ as $t \rightarrow \infty$ (see solution (4.13)) can enable the lower-layer vortex to break free from the lens. When the first term in (4.14) becomes comparable to the second one, the lower-layer particles can move away from the lens and balance the 'loss' of $h$ by a 'gain' in $\nabla^{2} \psi$. It should be emphasized though that, if the two terms of $P V_{2}$ are of the same order, our asymptotic equations are no longer applicable, as the previous scaling of $\psi$ becomes invalid. This question is discussed in more detail in $\S 4.4$ below.

Finally, consider the parameter range of Flierl (1984), $\alpha \gg \delta$, in which case the planetary vorticity term dominates (4.14). The PV-contours are no longer closed in the lower layer, hence the fluid is not trapped under the eddy and Rossby (planetary) waves can be effectively radiated.

(ii) Physically, the most unexpected result is that the meridional component of the translation speed rapidly 'overtakes' the zonal component (see figure 7). The former 


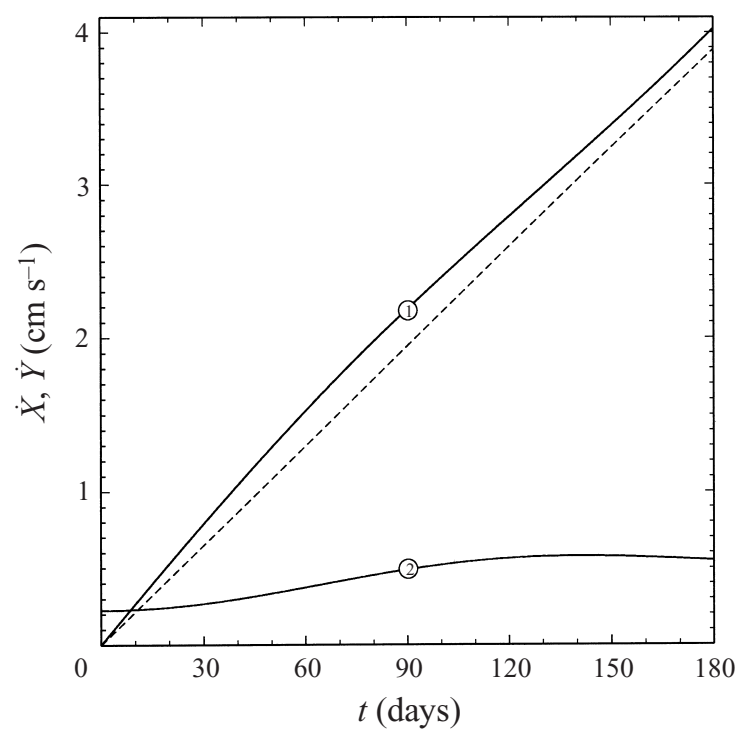

FiguRE 7. The zonal (2) and meridional (1) components of the translation speed of a solid-body rotating lens with $H_{\max }=400 \mathrm{~m}, r_{0}=120 \mathrm{~km}$. The dashed line shows the large- $t$ asymptotic solution (4.13).

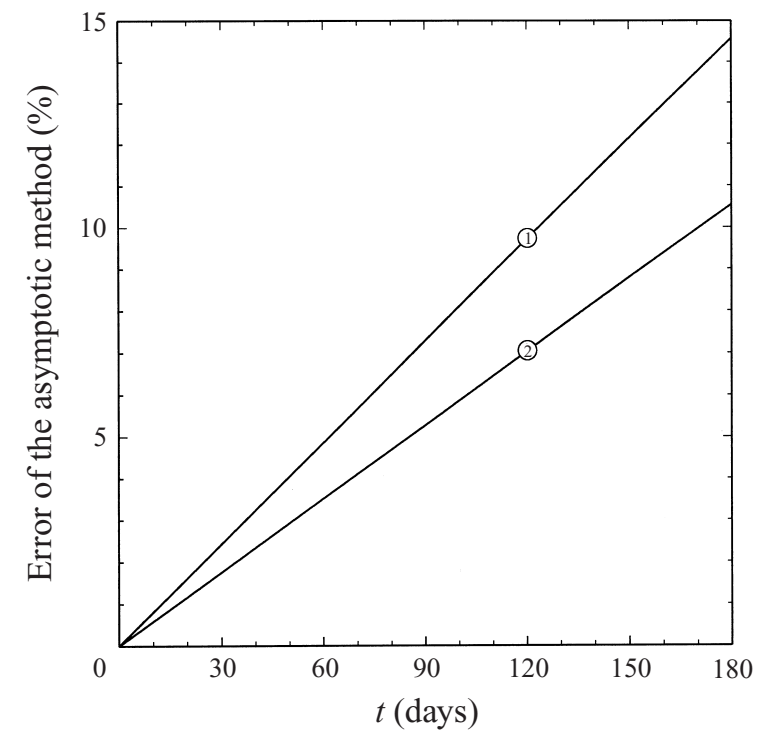

FIGURE 8 . The error of our asymptotic method for different solid-body rotating lenses:

(1) $H_{\max }=400 \mathrm{~m}, r_{0}=120 \mathrm{~km}$; (2) $H_{\max }=300 \mathrm{~m}, r_{0}=120 \mathrm{~km}$.

grows approximately linearly (in accordance with the large- $t$ solution (4.13)), whereas the latter oscillates about the Nof value. This question is discussed in more detail in $\S 4.4$ below.

(iii) The error of our asymptotic method accumulates relatively slowly (see figure 8). In order to understand how the error was measured, observe that the most 'dangerous' 


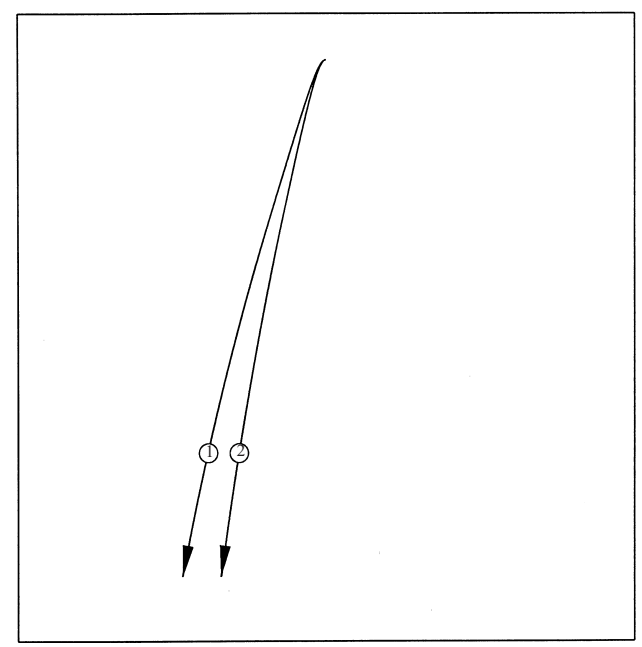

FIGURE 9. The trajectories of lenses of different shapes: (1) solid-body rotating lens (4.11); (2) differentially rotating lens (4.12).

terms omitted from the exact equations were

$$
\frac{\alpha^{2}}{\delta^{2}} \dot{Y} \frac{\partial \nabla^{2} \psi}{\partial y} \text { and } \frac{\alpha^{2}}{\delta^{2}} J\left(\psi, \nabla^{2} \psi\right)
$$

(both appear in equation (2.19)). According to the above large- $t$ solution, these terms are proportional to $t^{2}$, whereas the other terms omitted are proportional to $t$. In order to make sure that these terms are smaller than some of the terms retained, say

$$
\frac{\alpha}{\delta} \dot{Y} \frac{\partial h}{\partial y} \quad \text { and } \quad \frac{\alpha}{\delta} J(\psi, h)
$$

it is enough to ensure that

$$
|h| \gg \frac{\alpha}{\delta}\left|\nabla^{2} \psi\right|
$$

Thus, the error of our asymptotic method can be defined as

$$
\text { error }=\frac{\alpha}{\delta} \frac{\max \left\{\left|\nabla^{2} \psi\right|\right\}}{\max \{H\}}
$$

and, in our simulations, it has never exceeded $15 \%$ (over a period of 6 months of vortex evolution). It should also be observed that thinner vortices 'generate' error slower than thicker vortices (see figure 8).

(iv) Figure 9 shows the trajectories of lenses, and it is found that all lenses of profile (4.11), regardless of their thickness and size, fall onto curve (1), whereas all lenses described by (4.12) fall onto curve (2). Thus, given the same shape of the lens, its trajectory does not depend on its parameters-those affect only how far the lens travels along the trajectory. This property, in fact, follows from the invariance of our governing equations with respect to the following scaling transformation:

$$
r \rightarrow \gamma_{1} \widetilde{r}, \quad H \rightarrow \gamma_{2} \widetilde{H},
$$




$$
\begin{array}{ccc} 
& \frac{1}{2} r_{0}=55 \mathrm{~km} & \frac{1}{2} r_{0}=70 \mathrm{~km} \\
& H_{\max }=280 \mathrm{~m} & H_{\max }=380 \mathrm{~m} \\
V_{\max }=0.55 \mathrm{~m} \mathrm{~s}^{-1} & \Delta x=50 \mathrm{~km} & \Delta x=70 \mathrm{~km} \\
& \Delta y=145 \mathrm{~km} & \Delta y=235 \mathrm{~km} \\
& H_{\max }=385 \mathrm{~m} & H_{\max }=530 \mathrm{~m} \\
V_{\max }=0.85 \mathrm{~m} \mathrm{~s}^{-1} & \Delta x=70 \mathrm{~km} & \Delta x=125 \mathrm{~km} \\
& \Delta y=330 \mathrm{~km} & \Delta y=510 \mathrm{~km}
\end{array}
$$

TABLE 1. The zonal and meridional displacements $(\Delta x$ and $\Delta y)$ of oceanic lenses from their initial positions. The profile of the lenses is given by (4.12) (differential rotation); $V_{\max }$ and $H_{\max }$ are the maximum swirl velocity and thickness of the lens, respectively; $\frac{1}{2} r_{0}$ is the radius corresponding to $V_{\max }$. The displacements computed correspond to the period of 6 months. The top left cell models a particular Gulf Stream ring (WCR82B 4/82) observed by Olson (1991).

where $\gamma_{1,2}$ are arbitrary constants. Correspondingly, the mass of the lens transforms as

$$
M \rightarrow \gamma_{1}^{2} \gamma_{2} \widetilde{M}
$$

It is impossible, however, to derive a similar formula for the net angular momentum of the lens (its transformation is not scaling-like), so we have to introduce an independent coefficient $\gamma_{3}$ for it:

$$
A \rightarrow \gamma_{3} \widetilde{A}
$$

All three arbitrary constants, $\gamma_{1,2,3}$, can be eliminated from the governing equations by the transformation

$$
t \rightarrow \frac{1}{\gamma_{2}} \widetilde{t}, \quad \dot{X} \rightarrow \frac{\gamma_{3}}{\gamma_{1}^{2} \gamma_{2}} \tilde{X}, \quad \dot{Y} \rightarrow \frac{\gamma_{3}}{\gamma_{1}^{2} \gamma_{2}} \tilde{\dot{Y}}, \quad P_{c, s} \rightarrow \frac{\gamma_{3}}{\gamma_{1} \gamma_{2}} \widetilde{P}_{c, s}
$$

Observe that $\dot{X}$ and $\dot{Y}$ transform in exactly the same way, which means that the trajectory of the lens remains the same for all values of $\gamma_{1,2,3}$.

(v) The results of our simulations show that sufficiently large and strong lenses are capable of travelling significant distances in the ocean (see table 1). Generally, stronger and larger lenses travel faster than weaker and smaller ones. It appears though that the important factors are the net angular momentum and mass, rather than the strength and thickness as such.

\subsection{Discussion}

There are two crucial questions associated with the physical interpretation of the results obtained: What causes the meridional drift of lenses? What is the impact of the growing dipolar flow on the lens?

(i) The strong meridional drift of lenses is caused, no matter how paradoxical this sounds, by their weak zonal drift. When the Nof mechanism forces the lens to move westwards, it creates a zonal gradient in the pressure of the lower layer (an increase of pressure in front of the lens and a decrease behind). In turn, the pressure gradient gives rise to a geostrophic flow in the lower layer. Given the sign of the gradient, this flow is directed equatorwards, and it carries the lens with it.

Observe that the meridional translation of the lens does not create a pressure gradient in the lower layer as, in this direction, the lens goes with the lower-layer flow. A pressure gradient can be created only if the lens moves 'by itself' (through Nof's mechanism) and thus 'pushes' the lower-layer particles. 
The fact that the meridional translation accelerates with $t$ can be explained using equations (3.31) and (3.34). The latter links the meridional speed with the coscomponent $P_{c}$ of the streamfunction, and the former indicates that the growth rate of $P_{c}$ is proportional to the zonal speed $\dot{X}$. Since the zonal speed is, roughly, constant, this suggests that $P_{c}$ (and $\dot{Y}$ ) grow, roughly, linearly. In other words, the meridional acceleration of the lens is proportional to its zonal speed.

The possibility of strong meridional movement of eddies in a two-layer fluid has been discussed by Dewar \& Gailliard (1994). It should be noted, however, that the meridional motion in that case was caused by a strong deep flow included in the model (whereas, in the present paper, the lower layer is initially at rest). The conclusions of both papers qualitatively agree with Armi et al. (1989), who tracked a Mediterranean salt lens in the Atlantic and showed that its trajectory is, mainly, meridional-but there is no evidence indicating which effect is responsible for it. On the other hand, Chassignet \& Cushman-Roisin (1991) observed (numerically) almost exclusively zonal translation of eddies, which can possibly be accounted for by the zonal wind included in their model.

(ii) A physical interpretation of the growing dipolar flow is much more unclear so the second question is less straightforward to answer than the first.

In the one-layer case, one is left with no other possibility but to assume that the vortex loses its radial symmetry and evolves into a fully asymmetric patch (it is clear, of course, that the growing dipolar flow will generate a quadrupolar circulation, etc.). Our multi-layer problem is somewhat less clear, as the dipolar solution may saturate at a finite, albeit relatively large, level. Indeed, in contrast to the one-layer case, the two-layer problem has a parameter which could 'set' this level, namely $\delta$. Accordingly, recall that the lower-layer streamfunction was scaled by $\alpha$ (see (2.15)) - which turned out to be too small as $t \rightarrow \infty$ and should therefore be replaced by

$$
\Psi_{2}=\delta \frac{g^{\prime} H_{1}}{f} .
$$

The meridional component of the translation speed should also be rescaled as

$$
C_{y}=\delta \sqrt{g^{\prime} H_{1}},
$$

whereas the zonal component should remain the same:

$$
C_{x}=\alpha \sqrt{g^{\prime} H_{1}}
$$

(recall that $\dot{X}$ does not grow with $t$ ). Next, the growth becomes important only as $t \rightarrow \infty$; therefore, the scaling of $t$ should also be 'extended':

$$
T=\alpha^{-1} f^{-1}
$$

(compare this with the old scaling (2.13)). The rescaled version of equations (2.16)(2.19) is

$$
\begin{gathered}
{\left[\alpha\left(\frac{\partial}{\partial t}-\dot{X} \frac{\partial}{\partial x}\right)-\delta \dot{Y} \frac{\partial}{\partial y}\right] u+u \frac{\partial u}{\partial x}+v \frac{\partial u}{\partial y}+\frac{\partial}{\partial x}(h+\delta \psi)=(1+\alpha y) v,} \\
{\left[\alpha\left(\frac{\partial}{\partial t}-\dot{X} \frac{\partial}{\partial x}\right)-\delta \dot{Y} \frac{\partial}{\partial y}\right] v+u \frac{\partial v}{\partial x}+v \frac{\partial v}{\partial y}+\frac{\partial}{\partial y}(h+\delta \psi)=-(1+\alpha y) u,} \\
{\left[\alpha\left(\frac{\partial}{\partial t}-\dot{X} \frac{\partial}{\partial x}\right)-\delta \dot{Y} \frac{\partial}{\partial y}\right] h+\frac{\partial}{\partial x}(h u)+\frac{\partial}{\partial y}(h v)=0,}
\end{gathered}
$$




$$
\left[\frac{\alpha}{\delta}\left(\frac{\partial}{\partial t}-\dot{X} \frac{\partial}{\partial x}\right)-\dot{Y} \frac{\partial}{\partial y}\right]\left(h+\nabla^{2} \psi\right)+\frac{\alpha}{\delta} \frac{\partial \psi}{\partial x}+J\left(\psi, h+\nabla^{2} \psi\right)=0 .
$$

Unfortunately, this set is much more complicated than the original equations and, at the moment, it is unclear how to analyse it. On the other hand, it is needed only for a description of long-term (longer than 6 months) vortex evolution. At this timescale, however, near-surface vortices are affected by external currents, bottom topography, wind, etc., and the whole approach has to be revised.

\section{Conclusion}

In this section, we recall the main results obtained in the present work. We were concerned with near-surface vortices in a two-layer fluid on the beta-plane. Initially, vortices are radially symmetric and localized in the upper layer. Two non-dimensional parameters govern their evolution and translation: the non-dimensional beta-effect number $\alpha$ (given by (1.1)) and the ratio $\delta$ of the thickness of the vortex to the total depth of the fluid (given by (1.2)). As suggested by oceanic observations, we considered the case $\alpha \ll \delta \ll 1$. It turned out that, in this limit, the beta-term in the lower-layer equation is negligible, which indicates physically that the effect of barotropic Rossby-wave radiation on the dynamics of the vortex is weak.

Using a suitable asymptotic technique, we considered the most realistic case, $\alpha \ll \delta$, and concentrated mainly on anticyclonic eddies with compact support and lenses, for which the thickness $H(r)$ of the vortex satisfies the condition

$$
\frac{\mathrm{d} H}{\mathrm{~d} r}=0 \quad \text { for } \quad r \geqslant r_{0} .
$$

The following features were observed:

(i) The meridional (equatorward) component of the translation speed of the vortex rapidly 'overtakes' the zonal (westward) component. The former grows approximately linearly, whereas the latter oscillates about the Nof (1981) value (i.e. about the speed of translation of a vortex in a one-layer fluid). The growth of the meridional component has a simple physical explanation based on the pressure difference in the lower layer in front of and behind the lens (see $\$ 4.4$ ).

(ii) Vortices of the same shape, but different radii and amplitudes, follow the same trajectory. The amplitude and radius affect only the absolute value, but not the direction, of the translation speed.

(iii) In the lower layer below the vortex, a 'region' is generated where the velocity of the fluid is growing linearly with time. The velocity field in this region becomes more and more homogeneous (and equal to the translation speed of the vortex).

The last conclusion is easier to understand if we observe that, even though the initial velocity field of the vortex is localized in the upper layer, the potential vorticity has a strong anomaly in the lower layer below the vortex (due to the displacement of the interface). As the displacement translates along with the vortex, the lower-layer particles must follow the vortex too-otherwise they cannot preserve their initial PV values. This argument appears to imply that the vortex 'drags' the lower-layer particles with it; however, one can look at it the other way around and assume that the flow of particles in the lower layer is what moves the vortex along its trajectory.

I would like to thank G. M. Reznik for stimulating discussions. 


\section{REFERENCES}

Armi, L., Hebert, D., Oakey, N., Price, J. F., Richardson, P. L., Rossby, H. T. \& Ruddick, B. 1989 Two years in the life of a Mediterranean salt lens. J. Phys. Oceanogr. 19, 354-370.

Benilov, E. S. 1996 Beta-induced translation of strong isolated eddies. J. Phys. Oceanogr. 26, 2223-2229.

Chassignet, E. P. \& Cushman-Roisin, B. 1991 On the influence of a lower layer on the propagation of nonlinear oceanic eddies. J. Phys. Oceanogr. 21, 939-957.

Dewar, W. K. \& Gailliard, C. 1994 The dynamics of barotropically dominated rings. J. Phys. Oceanogr. 24, 5-29.

FLIERL, G. R. 1984 Rossby wave radiation from a strongly nonlinear warm eddy. J. Phys. Oceanogr. 14, 47-58.

Flierl, G. R., Stern, M. E. \& Whitehead JR, J. A. 1983 The physical significance of modons: Laboratory experiments and general integral constraints. Dyn. Atmos. Oceans 7, 233-263.

NoF, D. 1981 On the $\beta$-induced movement of isolated baroclinic eddies. J. Phys. Oceanogr. 11, $1662-1672$.

Olson, D. B. 1991 Rings in the ocean. Ann. Rev. Earth Planet. Sci. 19, 283-311.

ReZnik, G. M. \& DeWAR, W. K. 1994 An analytical theory of distributed axisymmetric barotropic vortices on the $\beta$-plane. J. Fluid Mech. 269, 310-321.

SutYrin, G. G. \& Dewar, W. K. 1992 Almost symmetric solitary eddies in a two-layer ocean. J. Fluid Mech. 238, 633-656.

Sutyrin, G. G. \& Flierl, G. R. 1994 Intense vortex motion on the beta plane: Development of the beta gyres. J. Atmos. Sci. 51, 773-790.

Swaters, G. E. 1998 Dynamics of radiating cold domes on a sloping bottom. J. Fluid Mech. 364, $221-251$ 